\title{
The Influence of Higher Education Teachers' Professional Self-efficacy and Emotion Regulation Method to the Subjective Emotional Experience
}

\author{
Zhang Yuan \\ Department of Education and Science, Shanxi University, Taiyuan, Shanxi (331675842@qq.com)
}

\begin{abstract}
Emotional regulation research is an important front hotspot of the emotional studies in recent years as well as the occupation self-efficacy of teachers. This research is a combination of these two aspects, which is from the different classification of emotion regulation method to the standard of self-efficiency of the college teacher. Through the experiment, it will be systematically investigated the effect of emotional experience to college teachers from the high self-efficiency, low self-efficiency and diverse emotion regulation methods. The research result indicates that firstly, there will be different effect of emotional subjective feelings if it has various emotion regulation methods. Evaluation of neglect will effectively weaken the subjective feelings of negative emotionality. Besides, evolution of value will enhance the subjective feelings of negative emotionality. Secondly, the difference of occupation self-efficiency level will lead a disparate impact of negative emotion subjective perception and high efficiency has little influence to negative emotion. It is efficiency to induce certainly intensive negative emotions according to laboratory technology of emotions evoking. 4. It has no relationship with teacher professional self-efficacy levels and gender. 5. The interactive effect between self-efficacy level and emotion regulation style is not obvious.
\end{abstract}

Key words-Emotion regulation Self-efficacy Teachers of higher education Experiment

\section{高职教师不同职业自我效能与不同情绪调节方式对主 观情绪体验的影响 \\ 张媛 \\ 山西大学教育科学学院, 太原, 山西, 中国}

摘 要 情绪调节研究是近年情绪研究领域中一个重要的前沿热点。教师职业自我效能也是研究教师心理的重要领域, 本研究将两 者相结合, 从情绪调节方式的不同和高职教师自我效能水平不同的分类出发, 通过前后测实验, 系统地探讨了高效能感、低效能感和 不同情绪调节方式（评价重视、评价忽视）对高职教师主观情绪体验的影响, 研究结果表明: 1 教师职业自我效能水平与性别无关; 2 不同情绪调节方式对情绪主观感受的影响不同: 评价忽视能有效减弱负情绪的主观感受; 评价重视能有效增强负情绪的主观感受; 3 不同职业自我效能水平对负性情绪主观感受的影响不同, 高效能感水平受到负性情绪的影响较小; 4 情绪调节方式与自我效能水平两 者交互作用不明显。

关键词 高职教师 职业自我效能 情绪调节方式 情绪主观体验 实验研究

1. 问题的提出

教师的情绪是学生学习的重要影响因素之一。在我 国新一轮教学改革关注学生发展的背景下, 对教师的教
学工作行为进行研究显得尤为重要。教师的自我效能感,

一方面可能会影响到教师教学行为, 另一方面也影响学 生的自我效能和学习效果。自我效能高的教师对教学投 
入热情较高, 能够不断钻研, 改革进取, 采取各种有效 方法组织教学, 而自我效能低的教师则相反。教师的工 作态度和方式也必然影响学生的精神面貌、学业效果。 教师的自我效能感也影响教师的身心健康和职业发展。 自我效能感低的教师常常在教学中付出沉重的代价而教 学效果不佳。有研究表明职业效能感高的教师对学生的 学习及创造能力有积极的影响, 教师的情绪对学生的学 习和发展也有明显的影响。除了从课堂教学策略及教学 能力因素开展研究之外, 教师的职业自我效能及情绪调 节方式也是一种值得关注的取向。教师的自我效能不仅 是教学效能感, 还具有职业自我效能感, 对教师的工作 也有一定的影响作用, 但就现有研究而言, 对于教师的 职业自我效能的研究还处在初级阶段。我国国内对教师 自我效能感的研究还不太多, 主要是对影响教师自我效 能感因素的探讨。辛涛等人 (1994) 研究发现, 学校制度 的完整性、工作提供的发展条件、学校的支持系统、校 风、教师关系、师生关系等 6 类因素与教师的个人教学 效能感之间存在显著的正相关; 俞国良等人 (1995) 研究 发现, 随着教龄的增加, 教师的一般教学效能感呈下降 趋势, 而个人教学效能感表现出上升的倾向; 在校大学生 和中学教师在教学效能感两个维度上存在着显著差异; 性别和学历因素对教师教学效能感不存在显著影响。另 外王才康等 2001 年研究了幼儿教师自我效能感与焦虑 水平的关系, 结果发现教师的自我效能感和状态焦虑及 特质焦虑量负相关, 这说明教师自我效能感还与教师的 心理健康有密切的关系。

根据已有研究, 我们可以得出以下结论不同情绪调 节方式对情绪主观感受的调节程度及调节后果影响显著 (李静); 在大学生样本中分心是最频繁提到的情绪调节 方式 (Larsen); 儿童的情绪困扰与自我效能感密切相关 (Michael Newlin); 某些能力较高的人将会才需一些更 具有建设性的方式来调节情绪; 情绪调节和自我效能可 以共同帮助经历失败的个体尽快有效地恢复（Alan D. Boss and Henry P. Sims Jr)。由此我们可以推断, 自我效能与情绪调节之间关系非常密切, 研究二者之间 的关系对于充实理论与指导实践都具有重要意义, 本研 究立足于情绪调节方式的研究, 将自我效能与情绪调节 方式相结合, 研究二者的关系。此外, 现有研究多以学 生为研究对象, 而对教师特别是高职教师情绪调节及职 业自我效能的研究尚不多见, 本文旨在研究高职教师职 业自我效能与情绪调节方式对其主观情绪感受的影响, 以此研究探索适合高职教师的合理情绪调节方式。

\section{2. 实验研究一一不同自我效能与情绪调节方式对主 观情绪体验的影响}

本实验主要研究情绪的诱发及情绪调节的执行, 探 讨不同职业自我效能水平以及不同的情绪调节方式对情 绪主观感受的影响。实验中的情绪调节方式分别是评价 忽视、评价重视与表情抑制的结合, 其中, 评价忽视和 评价重视属原因调节, 表情抑制属反应调节, 最为本实 验的控制变量。

\section{1 研究目的与假设}

研究目的: 本实验为研究的第一部分, 通过实验手 段来诱发所要研究的具有一定强度的情绪, 并对这些情 绪采用不同的情绪调节方式进行调节, 以没有进行情绪 调节的控制组作为参照, 主要考察不同情绪调节方式对 情绪主观感受的影响及不同情绪调节方式的执行情况。

研究假设: 不同情绪调节方式和不同效能感对情绪 主观感受的影响不同。

\section{2 研究方法}

\subsection{1 被试}

来自山西职业技术学院教师 100 名, 其中男教师 49 名, 女教师 51 名, 平均年龄 32.90 岁, $\mathrm{SD}=7.015$ 。

\subsection{2 实验设计}

本研究为实验室实验法, 采用两因素两水平实验设 计。以区组方法将被试分成两组, 分别接受 2 种实验条 件: 评价忽视、评价重视。实验中的自变量为教师职业 自我效能水平与情绪调节方式 (原因调节), 通过效能测 验分组、情绪调节方式的指导语实现, 因变量为情绪主 观感受的变化。控制变量为反应调节 (全部为表情抑制)

\section{2 .3 情绪诱发材料}

在收集分析了国内外同行曾经研究和使用的情绪诱 发材料及市场上可能有的情绪音像材料后, 在情绪诱发 预实验的初步检验基础上，选取影片《妈妈再爱我一次》 里的“母子分离”片段作为情绪诱发材料, 长度为 4 分 34 秒。

\subsection{4 问卷}

教师职业自我效能问卷: 问卷为 5 点量表 (李卫星, 2009), 以评定教师的职业自我效能水平, 以此作为被试 篮选分组的依据, 为下面研究做准备。 
情绪报告表: 在分析了由 Ekman 等人最初制作 Gross 等人多次使用的情绪报告表的基础上，本实验选取了 7 个情绪形容词为主观报告内容, 构成一个从 0（没有） 到 5 (极度强烈) 的 6 个等级量表, 以评定情绪主观感 受的强度。本研究在预实验的基础上选取了快乐、兴趣 2 种正情绪和紧张、恐惧、厌恶、愤怒、痛苦 5 种负情 绪。实验主要研究的是负情绪的诱发和调节, 选取快乐、 兴趣 2 种正情绪是为了避免负情绪在前测中的先导作 用。情绪报告表包括前测和后测两部分

\section{2 .5 实验程序}

事先召集被试, 被试以自愿的形式报名参加, 实验 前通过自我效能测试选择合适的被试, 即分数处在整体 前后 $27 \%$ 排名的教师, 将两端被试分别随机分为两组并 混合。告知所选被试接下来的实验是为了对人类的情感 和认知的关系有更多地了解, 并预约具体实验时间。实 验分两天进行, 第一天篎选合适的被试, 第二天将符合 条件的被试按照量子变量的不同组合分为 4 组, 每组为 一种情绪调节方式和不同效能水平的组合, 分别接受实 验, 每组试验时间大约 40 分钟。实验以小组形式集体进 行, 每组 15 名被试。后期剔除极端之后剩余 58 名被试。

实验人员为主试 2 名, 1 名主要负责整个实验流程, 1 名负责分发、收集问卷, 实验后的情绪安抚工作及处 理突发事件, 如被试中途退出。实验在带有情绪诱发材 料呈现系统的实验室进行, 类似于一个小型电影厅。

实验前, 先告知被试实验的大致情况, 在自愿的前 提下填写有关个人资料。实验依次分 4 组进行, 每组采 用一种情绪调节方式与一种效能感水平的组合。两组依 次为高效能感、评价重视, 高效能感、评价忽视, 低效 能感、评价重视, 低效能感、评价忽视的结合。在观看 前, 主试告知被试 “现在让自己保持平静, 什么也不要 想, 可以做几个深呼吸让自己平静下来。” 1 分钟后被试 填写情绪报告表一 (前测), 报告他们在观看片段前的情 绪感受强度, 在情绪报告表一的最后呈现指导语, 主试 告知被试 “现在将指导语默念三遍, 以确保自己在指导 语的指示下观看下面的电影片段。”指导语呈现 1 分钟后 播放电影片段, 影片时长 8 分钟。

指导语系列:

评价忽视组: 下面将播放一段影片, 请您认真仔细 地观看, 以客观、理智的态度来对

待影片, 告诉自己这只是电影技术而已, 不是真的, 尽量不去感受影片中的情绪。在观看的同时, 不要将您 的感受表露出来, 尽量掩饰您的表情, 不要让别人看出
您的情绪感受。

评价重视组: 下面将播放一段影片, 请您认真仔细 地观看, 尽可能将自己置身于影片

中, 想象着这些事件就发生在自己身上一样, 并充 分感受影片中的情绪。在观看的同时，不要将您的感受 表露出来, 尽量掩饰您的表情, 不要让别人看出您的情 绪感受。

观看结束后, 被试填写情绪报告表二 (后测), 报告 他们在观看过程中及观看结束后填写后测问卷前这二个 阶段的情绪感受强度。

\subsection{6 数据收集与处理}

实验数据由情绪报告表一、情绪报告表二组成, 收 集后使用 SPSS11. 5 for Windows 统计软件包进行分析。

\section{3. 结果与分析}

\section{1 情绪的主观感受}

对观看前所有被试情绪主观体验得分在 4 个实验组 间是否存在差异进行方差检验, 结果显示, 4 个实验组 情绪均未产生显著差异, 说明被试的情绪初始状态是一 致的，可认为被试来源于相同的总体。

处理前总分

\begin{tabular}{|c|c|c|c|c|c|}
\hline & Sum of Squares & df & Mean Square & F & Sig. \\
\hline 组间 & 7.140 & 3 & 2.380 & .592 & .623 \\
\hline 组内 & 216.929 & 54 & 4.017 & & \\
\hline 总数 & 224.069 & 57 & & & \\
\hline
\end{tabular}

\section{2 结果分析}

\subsection{1 情绪的诱发情况}

本研究通过实验室电影片段成功的诱发了研究所需 要的负情绪紧张、恐惧、厌恶、愤怒和痛苦, 显著增强 了这些负情绪的主观感受; 减弱了正情绪快乐和兴趣的 主观感受。这表明采用实验室实验的手段, 选取适当的 情绪诱发材料能有效诱发具有一定强度的情绪, 再次验 证了实验室情绪诱发技术的有效性, 这种实验手段是诱 发情绪的一种有效方法。

\begin{tabular}{|c|c|c|c|c|c|}
\hline & I & Vlin & Iax & IIII & Std. Deviation \\
\hline 处理前总分 & 58 & 0 & 20 & 10.12 & 5.178 \\
\hline 处理后总分 & 58 & .00 & 7.00 & 1.58 & 1.98 \\
\hline
\end{tabular}


3.2.2 情绪调节方式与职业自我效能的交互作用分析

组前后分差进行多因素方差分析, 结果如下:

\begin{tabular}{|c|c|c|c|c|c|}
\hline Source & $\begin{array}{c}\text { Type III Sum } \\
\text { of Squares }\end{array}$ & df & $\begin{array}{c}\text { Mean } \\
\text { Square }\end{array}$ & F & Sig. \\
\hline 职业效能 & 209.393 & 1 & 209.393 & 11.470 & .001 \\
\hline 调节方式 & 398.457 & 1 & 398.457 & 21.827 & .000 \\
\hline $\begin{array}{c}\text { 职业效能 } \\
\text { 调节方式 }\end{array}$ & 23.324 & 1 & 23.324 & 1.278 & .263 \\
\hline Total & 5837.000 & 58 & & & \\
\hline $\begin{array}{c}\text { Corrected } \\
\text { Total }\end{array}$ & 1612.431 & 57 & & & \\
\hline
\end{tabular}

从表中数据可以看出两种作用的交互作用不明显, 所以以下对两自变量的作用分别作出分析。

\subsection{3 两自变量各自对因变量影响的分析}

\subsubsection{1 情绪调节对主观感受变化的影响}

通过对前后测主观情绪体验的分差进行比较发现, 在观看中即情绪调节进行的过程中, 评价忽视降低了负 性情绪的主观感受, 即分差变化较小 $(M=5.8, S D=5.09)$; 评价重视增强了负性情绪的主观感受, 即分差变化较大 $(M=11.07, S D=4.21)$; 对不同调节方式两组进行单因素 方差分析可知两组的分差异显著 $(p<0.05)$, 说明不同情 绪调节方式对负性情绪的主观感受产生了不同影响, 评 价忽视较为有效。

前后分差

\begin{tabular}{|c|c|c|c|c|c|}
\hline & 人数 & 平均分 & 标准差 & Minimum & Maximum \\
\hline 评价忽视 & 28 & 5.8214 & 5.09214 & -2.00 & 19.00 \\
\hline 评价重视 & 30 & 11.0667 & 4.20946 & 3.00 & 20.00 \\
\hline Total & 58 & 8.5345 & 5.31867 & -2.00 & 20.00 \\
\hline
\end{tabular}

前后分差

\begin{tabular}{|c|c|c|c|c|c|}
\hline & $\begin{array}{c}\text { Sum of } \\
\text { Squares }\end{array}$ & df & $\begin{array}{c}\text { Mean } \\
\text { Square }\end{array}$ & F & Sig. \\
\hline Between Groups & 398.457 & 1 & 398.457 & 18.381 & .000 \\
\hline Within Groups & 1213.974 & 56 & 21.678 & & \\
\hline Total & 1612.431 & 57 & & & \\
\hline
\end{tabular}

\subsubsection{2 教师职业效能感高低比较}

通过对前后测主观情绪体验的分差进行比较发现, 在观看中即情绪调节进行的过程中, 高效能感教师降低 了负性情绪的主观感受, 即分差变化较小 $(\mathrm{M}=6.65$,
$\mathrm{SD}=4.79$ ); 评价重视增强了负性情绪的主观感受, 即分 差变化较大 $(\mathrm{M}=10.41, \mathrm{SD}=5.23)$; 对不同调节方式两 组进行单因素方差分析可知两组的分差异显著 $(\mathrm{p}<0.05)$, 说明不同职业自我效能水平对负性情绪的主 观感受产生了不同影响, 高效能感受引发负性情绪影响 较小。

\begin{tabular}{|c|c|c|c|c|c|}
\hline & 人数 & 平均数 & 标准差 & Minimum & Maximum \\
\hline 低效能感 & 29 & 10.4138 & 5.22711 & .00 & 20.00 \\
\hline 高效能感 & 29 & 6.6552 & 4.79044 & -2.00 & 16.00 \\
\hline Total & 58 & 8.5345 & 5.31867 & -2.00 & 20.00 \\
\hline
\end{tabular}

\begin{tabular}{|c|c|c|c|c|c|}
\hline & $\begin{array}{c}\text { Sum of } \\
\text { Squares }\end{array}$ & df & $\begin{array}{c}\text { Mean } \\
\text { Square }\end{array}$ & F & Sig. \\
\hline Between Groups & 204.845 & 1 & 204.845 & 8.150 & .006 \\
\hline Within Groups & 1407.586 & 56 & 25.135 & & \\
\hline Total & 1612.431 & 57 & & & \\
\hline
\end{tabular}

3.3 结论

\subsection{1 教师职业自我效能水平与性别无关}

3.3.2 通过实验室情绪诱发技术能有效诱发一定强度的 负情绪

3.3.3 不同情绪调节方式对情绪主观感受的影响不同: 评 价忽视能有效减弱负情绪的主观感受; 评价重视能引发 更强烈的负性情绪的主观感受

3.3.4 不同职业自我效能水平对负性情绪主观感受的影 响不同, 高效能感水平受到负性情绪的影响较小

3.3.5 情绪调节方式与自我效能水平两者交互作用不明 显

\section{4. 讨论}

\section{1 情绪的诱发情况}

本研究通过实验室电影片段成功的诱发了研究所需 要的负情绪, 显著增强了这些负情绪的主观感受; 减弱 了正情绪快乐和兴趣的主观感受。这表明采用实验室实 验的手段, 选取适当的情绪诱发材料能有效诱发具有一 定强度的情绪, 再次验证了实验室情绪诱发技术的有效 性，这种实验手段是诱发情绪的一种有效方法。

\section{2 评价忽视和评价重视的比较}

在原因调节中, 评价忽视有效降低了负性情绪的主 
观感受, 而评价重视对负性情绪主观感受的降低程度显 著低于评价忽视组。这表明原因调节中的减弱调节（评 价忽视）能有效降低负情绪的主观感受, 原因调节中的 增强调节 (评价重视) 调节效果则不佳。本研究符合原 因调节对情绪主观感受调节效果差异显著的观点, 与前 人的研究结果基本一致。

\section{3 高职业自我效能与低职业自我效能受负性情绪影响}

对于负性情绪的诱发, 高职业自我效能的教师收到 的影响显著低于地职业自我效能的教师，这与 “高职业 自我效能感的教师无论对学生还是对自身工作, 都会起 到正性的影响。”这一结论相对应。表明高职业自我效能 可以帮助高职教师有效减少负性情绪或其他负性影响的 影响。

\section{4 研究结果的现实意义}

本次实验主要探讨了不同的情绪调节方式以及不同 水平的职业自我效能对高职教师面对负性影响时应对的 有效性, 研究表明, 高职业自我效能的教师可以更为有 效地应对负性影响, 但无论效能感水平如何, 采用科学 正确的调节策略对其负性情绪的调节都能够产生有效的 帮助, 因此教师可以经过学习, 培养良好的情绪调节方 式, 以此来帮助自己更为有效地排解负性事件对自己产 生的不良影响。

\section{5 问题与不足}

\subsection{1 对本研究的深入:}

本研究采用单一的电影片段作为情绪诱发材料, 引 起了一定强度的情绪, 考察了对这一强度情绪的不同方 式的调节对记忆的影响。由于引发的情绪较为单一, 因 此对其他情绪不具有推广性。

\subsection{2 对情绪调节指标的拓展:}

情绪调节是通过一定的调节机制和策略, 使情绪在 主观感受、表情行为和生理反应等方面发生一定的变化。 本研究通过情绪主观感受的变化来考察不同方式的情绪 调节。对情绪调节的全面考察除了情绪感受的主观报告 外, 还可以对表情行为进行编码、记录和分析以及采用 生理多导仪等先进仪器对情绪调节的生理反应进行记录 和分析。

\subsection{3 对实验工具的改进:}

本文通过使用简单的情绪诱发视频对被试进行了负 性情绪的诱发, 在播放过程中由于被试相对集中, 因此 相互之间会产生一定的影响。对情绪的诱发除了使用视 频之外, 还可以使用文字材料, 现实情境等进行诱发, 在呈现方式上也可以采用独立电脑呈现以及使用先进软 件进行, 以此来代替简单的视频播放的方式。

\section{参考文献(References)}

1. Izard, C.E. The Psychology of Emotions. New York, Plenum, 1991

2. Gross JJ. Emotion regulation in adulthood: Timing is everything. Current Direction in Psychological Science, 2001, 10:214-219

3. Alan D. Boss Henry P. Sims Jr. Using emotion regulation and self-leadership for recovery Journal of Managerial Psychology Vol.23 No.2,2008 pp.135-150

4. Bandura A. Self-efficacy: Toward a unifying theory of behavior change. Psychological Review, 1977, 84:191- 215

5. Bandura A. The social foundation of thought and action. Englewood Cliffs, Prentice- Hall.1986

6. Li Jing, Lu Jiamei Different Emotional Regulation's Influence on Memory Acta Psychological Sinica 2007, 39(6); 1084 1092

7. Chen Xiaoling Emotion, Emotional Regulation's Influence on Self-control. Capital Normal University Master Essay, 2007, 34.

8. Huang Miner, Guo Dejun The substance of Emotional Regulation. Psychological Science, 2000, 23(1):109-110.

9. Huang Miner The Trend and Method of Emotional Regulation Applied Psychology 2001,7(2),17-22

10. Fang Ping Research and Analyze on University Students' Emotional Regulation Research on Teacher and Education 2007.9 19(5)

11. Lu Tao, Li Min Recognition Emotional Regulation in China Journal of Liaoning Administration and Education College 2008,11, 25(11)

12. Gao Chunshen Self-efficacy Theory 2000,1

13. Wu Zengqiang Self-efficacy: A Positive Self Belief Psychological Science 2001,24(4)

14. Zhang Dingkun Self-efficacy Theory and Current Research Psychology Dynamic 7(1)

15. Di Min, Huang Xiting, Zhang Zhijie. Career Self-efficacy Journal of Southwest Normal University, 2003, 29(5):22-26. 